\title{
Malaria among Children in Suriname, South America
}

\author{
Mary-Ann Wagijo', Edward van Eer², Remy A. Hirasing ${ }^{3}$ \\ ${ }^{1}$ Health Sciences-Specialization International Public Health, Antwerp, Belgium \\ ${ }^{2}$ Medical Mission, Paramaribo, Suriname \\ ${ }^{3}$ Department of Public and Occupational Health, EMGO Institute for Health and Care Research, VU University \\ Medical Centre, Amsterdam, The Netherlands \\ Email: ra.hirasing@vumc.nl
}

Received 27 March 2016; accepted 5 June 2016; published 8 June 2016

Copyright (C) 2016 by authors and Scientific Research Publishing Inc. This work is licensed under the Creative Commons Attribution International License (CC BY). http://creativecommons.org/licenses/by/4.0/

\begin{abstract}
Malaria in children is a serious infectious disease. In Suriname, many children died due to malaria in the past. To prevent malaria in children, anti-malaria campaigns were executed. The last campaign was implemented from 2005 to 2010 . To develop a strategy for the future, the current prevalence and mortality rates are needed. Since 1955, all confirmed malaria cases were registered by the Medical Mission and this database was used to determine the prevalence rate. For the mortality rates we used the cause of death which was registered on death certificates. Since 2005 no children died due to malaria. Also the prevalence rate dropped dramatically from 7255 in 2001 to 10 in 2014. However, despite these great results, 10 children were still diagnosed with malaria and there is a need for continuous attention to bring this number down to zero. The mobile goldmine workers can impose a threat for a new malaria epidemic, if they spread the malaria infection in Suriname. Therefore, effective prevention measures and treatment are still needed.
\end{abstract}

\section{Keywords}

Malaria, Children, Suriname, Prevention

\section{Introduction}

Malaria is an infectious disease, caused by the blood parasite Plasmodium. These parasites are transferred in the blood of human and large mammals by Anopheles mosquitoes. The most common parasite which causes malaria in Suriname is Plasmodium falciparum [1]. Symptoms of malaria are fever, headache, shivers, joint pains, repeated vomiting, diarrhoea and others [2]. The infection can lead to damage of the organs like: the brains, lungs, 
kidneys and blood vessels. Cerebral malaria is the most serious complication and can lead to death of the patient [2]. In 2015, there were 214 million malaria cases worldwide [3]. According to the World Health Organisation (WHO), malaria incidence among populations at risk fell by 37\% globally, between 2000 and 2015. In the same period, malaria death rates decreased by $60 \%$ globally among the world population and by $65 \%$ among children under 5 [3].

Also in Suriname, a former colony of the Netherlands and part of the Guyana Shield, the mortality and morbidity of malaria were high, especially in the interior of Suriname. In Suriname, historically, two epidemic endemic areas were defined: the coastal belt and the interior, where 9.8\% of the 500,000 inhabitants live [4] [5]. Since 1957, different malaria campaigns were executed in Suriname [4]-[6]. In 1968, malaria was eliminated in the coastal belt of Suriname [7]. Despite the campaigns, malaria is still present in the interior and the malaria cases in the interior imply a threat for re-emergence of malaria in Suriname.

The Medical Mission (MM), a private (non-profit) primary health care organisation, is responsible for health care in the interior of Suriname. Since 1955, all confirmed malaria cases in the interior are registered and weekly reported to the MM headquarters by MM health workers [7]. Since 2010, no malaria campaigns were implemented in Suriname [1]. In order to develop effective interventions for children, we need to know the extent and seriousness of malaria in the interior of Suriname. We looked for existing, trustworthy and accurate data of malaria cases among children. The most reliable source of information, in the non-mobile population of the interior, is the database of the MM for the incidence and death certificates for mortality rates. The research questions of this study are: what is the trend in malaria mortality among children in Suriname? And what is the prevalence of malaria in Suriname among children? To measure the seriousness of malaria, mortality rates were used. For the prevalence the data collected by the health workers of the MM was used.

\section{Methods}

The study population consisted of children from 1 to 19 years old. The 0 years old children were excluded from this research, because there already is an ongoing research in this group. For this study the death certificates were used to gain insight in the mortality rates. In Suriname, a medical doctor fills out the death certificate, in which a distinction is made between natural death (disease or old age) and unnatural death (external causes, like: accidental injury or violence). The cause of death on the death certificate is coded according to the International Classification of Diseases and Related Health Problems (10 ${ }^{\text {th }}$ Revision) (ICD10), since 1995 . In our study the codes 850 - 854 were used. For the prevalence of malaria, the registration of data of the health assistants was used. The health assistants performed a rapid diagnostic test and a blood smear for the identification of the blood parasite, in all children with fever and malaria related symptoms. The diagnosis of malaria is based on the microscopic identification of the malaria parasite. These tests were executed by instructed and trained health assistants of the MM. The data that is provided by them is: the date of the blood collection, name of the patient, name of the clinic, gender, result of the malaria test and any therapy. Weekly, the health workers send the malaria data together with the blood smear slides to the coordination center in Paramaribo. There the blood smear slides are double-checked, by means of quality control. Since 1998 the malaria data is registered in an automated database and all data collected before was imported in this database.

\section{Results}

Mortality due to malaria among children, decreased from 7 deaths in 2001, to 0 deaths in 2014. From 2005 there were no deaths from malaria among children between 1 and 19 years old (Figure 1). This has been confirmed by different experts.

For the prevalence of malaria in children the registration of the health care workers was used. Table 1 shows that the number of malaria positive cases declined from 7255 in 2001 to 10 cases in 2014. However, malaria is still present among children in the interior. The total number of positive cases in the last three years was 31 . So despite the impressive decline in malaria incidence, there are still children getting infected with malaria.

\section{Discussion}

In 2001, 7 children died from malaria. Since 2005, no children died due to malaria in Suriname. Another impressive achievement is the dramatic decline in malaria incidence among children from 7255 in 2001 to 10 in 


\section{Malaria mortality among children}

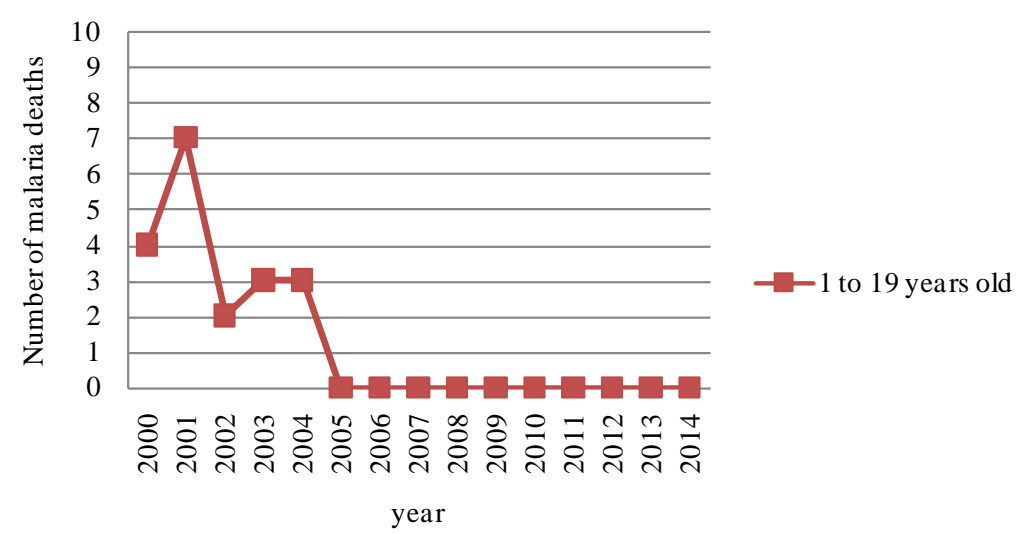

Figure 1. Malaria mortality among children in the interior of Suriname, from 2000 to 2014.

Table 1. Number of confirmed malaria cases from 2001 to 2014 in children in Suriname.

\begin{tabular}{|c|c|c|c|c|c|c|c|c|c|c|c|c|c|c|}
\hline \multirow{2}{*}{ Age in years } & \multicolumn{14}{|c|}{ Year } \\
\hline & 2001 & 2002 & 2003 & 2004 & 2005 & 2006 & 2007 & 2008 & 2009 & 2010 & 2011 & 2012 & 2013 & 2014 \\
\hline $1-4$ & 2946 & 2227 & 2585 & 1461 & 1489 & 461 & 44 & 38 & 18 & 7 & 5 & 3 & 1 & 0 \\
\hline $5-9$ & 1727 & 1456 & 1595 & 1025 & 1315 & 402 & 39 & 51 & 16 & 14 & 6 & 2 & 0 & 3 \\
\hline $10-14$ & 1392 & 1130 & 1219 & 714 & 815 & 227 & 23 & 29 & 12 & 8 & 3 & 2 & 2 & 5 \\
\hline $15-19$ & 1190 & 919 & 1001 & 555 & 665 & 187 & 31 & 20 & 20 & 10 & 6 & 7 & 4 & 2 \\
\hline Total & 7255 & 5732 & 6400 & 3755 & 4284 & 1277 & 137 & 138 & 66 & 39 & 20 & 14 & 7 & 10 \\
\hline
\end{tabular}

2014. This shows that fewer children were infected with the malaria parasite. The decrease in the mortality of malaria in children is due to the dramatic decline in incidence of malaria and/or better treatment in late 2003. The National Malaria Board introduced a new treatment in the interior, an artemisinin-based combination therapy. Before the new treatment, treatment against malaria was based on kinine [4] [7]. However, the decline in malaria cases and mortality was already noted before the introduction of new treatment.

Our results are broadly endorsed by several experts [7] and are not due to changes in registration. The significant decrease of malaria mortality is a fact, although the cause of the decrease is not specified or found yet. Throughout the years, different measures such as: the distribution of insecticide treated nets, active case detection campaigns, indoor residual spraying and health information about malaria, have been taken to eliminate or reduce the mortality, but the effects of these measures have not been investigated by RCTs. Therefore, we cannot conclude which specific measure or which combination of measures, led to the decrease. We can only speak of an association of circumstances and measures. The floods in the interior of Suriname in May 2006 are also mentioned as a cause for the decline [1]. However, the decline in malaria mortality was also seen before 2006.

A limitation of our study is the exclusion of 0 years old children in our target group. However, experts in Suriname reported that malaria incidence and mortality among 0 years is rare.

Currently, the biggest malaria challenge in Suriname regards the continuation of the achieved results. Malaria has a serious and high impact on public health, and it is important to prevent malaria also in Suriname. For this reason, there should be continuous attention for malaria, in order to maintain the current success. The threat of epidemic reintroduction of malaria in Suriname is real, since there are still people infected with malaria, also children. Furthermore, malaria is spread by the goldmine workers from countries where malaria prevalence is still high, predominantly from Brazil [1] [7]. The most promising and effective prevention will be a vaccine and currently there is one vaccine on the process of authorization and licensing. However, until this vaccine is worldwide available it is important to implement other effective preventive measures [7]. Monitoring malaria prevalence and mortality rates in Suriname remains valuable. 


\section{Conclusion}

The decrease of malaria mortality in children with malaria is due to the drastic decline in malaria incidence among children and/or the availability of new treatment since 2005. The threat of an increase in malaria mortality among children is present in Suriname, as there are still children infected with malaria and the goldmine workers from Brazil are still spreading the infection. Therefore, there is a continuous need of attention for malaria, especially for the children. The most promising measure against malaria is a vaccination and the development should be the main priority in the combat against malaria. However, until a vaccination becomes available we should use other effective prevention measures, such as insecticide treated nets and monitoring of the disease. Because malaria is not prevented in all children, early diagnosis and treatment are also very important.

\section{References}

[1] Hiwat, H., Mitro, S., Samjhawan, A., Sardjoe, P., Soekhoe, T. and Takken, W. (2012) Collapse of Anopheles darlingi Populations in Suriname after Introduction of Insecticide-Treated Nets (ITNs); Malaria down to near Elimination Level. The American Journal of Tropical Medicine and Hygiene, 86, 649-655. http://dx.doi.org/10.4269/ajtmh.2012.11-0414

[2] Center for Disease and Control. http://www.cdc.gov/malaria/about/index.html

[3] WHO (2015) World Malaria Report 2015. World Health Organization.

[4] Breeveld, F.J., Vreden, S.G. and Grobusch, M.P. (2012) History of Malaria Research and Its Contribution to the Malaria Control Success in Suriname: A Review. Malaria Journal, 11, 95. http://dx.doi.org/10.1186/1475-2875-11-95

[5] Rozendaal, J.A. (1990) Observations on the Distribution of Anophelines in Suriname with Particular Reference to the Malaria Vector Anopheles darlingi. Memorias do Instituto Oswaldo Cruz, 85, 221-234. http://dx.doi.org/10.1590/S0074-02761990000200014

[6] Barnes, S.T. and Jenkins, C.D. (1972) Changing Personal and Social Behaviour: Experiences of Health Workers in a Tribal Society. Social Science \& Medicine, 6, 1-15. http://dx.doi.org/10.1016/0037-7856(72)90003-0

[7] Hiwat, H., Hardjopawiro, L.S., Takken, W. and Villegas, L. (2012) Novel Strategies Lead to Pre-Elimination of Malaria in Previously High-Risk Areas in Suriname, South America. Malaria Journal, 11, 10.

http://dx.doi.org/10.1186/1475-2875-11-10 